\title{
Radio and $\gamma$-ray loud narrow-line Seyfert 1 galaxies in the spotlight
}

\author{
V. Karamanavis ${ }^{1, \star}$, E. Angelakis ${ }^{1}$, S. Komossa ${ }^{2}$, I. Myserlis ${ }^{1}$, \\ D. Blinov ${ }^{3}$ and J. A. Zensus ${ }^{1}$ \\ ${ }^{1}$ Max-Planck-Institut für Radioastronomie, Auf dem Hügel 69, D-53121 Bonn, Germany \\ email: vkaramanavis@mpifr.de \\ ${ }^{2}$ QianNan Normal University for Nationalities, Longshan Street, Duyun, Guizhou, China \\ ${ }^{3}$ Department of Physics and Institute of Theoretical and Computational Physics, \\ University of Crete, 71003 Heraklion, Greece
}

\begin{abstract}
Narrow-line Seyfert 1 (NLS1) galaxies provide us with unique insights into the drivers of AGN activity under extreme conditions. Given their low black hole (BH) masses and nearEddington accretion rates, they represent a class of galaxies with rapidly growing supermassive $\mathrm{BHs}$ in the local universe. Here, we present the results from our multi-frequency radio monitoring of a sample of $\gamma$-ray loud NLS1 galaxies ( $\gamma$ NLS1s), including systems discovered only recently, and featuring both the nearest and the most distant $\gamma \mathrm{NLS1s}$ known to date. We also present high-resolution radio imaging of $1 \mathrm{H} 0323+342$, which is remarkable for its spiral or ring-like host. Finally, we present new radio data of the candidate $\gamma$-emitting NLS1 galaxy RX J2314.9+2243, characterized by a very steep radio spectrum, unlike other $\gamma$ NLS1s.
\end{abstract}

Keywords. galaxies: active, galaxies: jets, galaxies: Seyfert, radio continuum: galaxies

\section{Introduction}

Narrow-line Seyfert 1 galaxies are a particular class of active galactic nuclei (AGN) that show: (i) small width of their broad optical emission lines, i.e. $\mathrm{FWHM}\left(\mathrm{H}_{\beta}\right) \leqslant 2000 \mathrm{~km} \mathrm{~s}^{-1}$ (Osterbrock \& Pogge 1985), pointing to a low black hole (BH) mass for these systems $\left(\sim 10^{6}-10^{8} \mathrm{M}_{\odot}\right)$, (ii) super-strong Fe II emission complexes, (iii) rapid X-ray variability, hinting that the broad-line region (BLR) and accretion disk are directly visible, (iv) near-Eddington accretion rates with ratios $L / L_{\mathrm{Edd}}$ between 0.1 and 1 (Boroson \& Green 1992), (v) super-soft X-ray spectra, and (vi) a multitude of intriguing multi-wavelength properties (see review by Komossa 2008).

There exists also an interesting small fraction of their population being radio-loud, featuring relativistic jets, and detected at $\gamma$ rays by Fermi $(\gamma$ NLS1s; Komossa et al. 2006; Abdo et al. 2009a,b). These few sources, the radio- and $\gamma$-ray-loud population, are exceptional because they show blazar-like observational attributes such as flat radio spectra, high brightness temperatures $\left(\mathrm{T}_{\mathrm{b}} \sim 10^{10}-10^{14} \mathrm{~K}\right)$, Doppler boosting, $\gamma$-ray emission and in few cases, one-sided jets (D'Ammando et al. 2013; Angelakis et al. 2015; Karamanavis 2015; Fuhrmann et al. 2016). However, possessing non-blazar physical properties, especially almost two orders of magnitude lower BH masses and high accretion rates. In this context, they constitute a source of new insights, able to give us clues on the formation of extragalactic jets and their evolution under conditions of high accretion rate and in a regime that is not probed by classical blazars. As possible explanations for their appearance, different scenarios have been proposed. Do they represent a young AGN population that rapidly grow their BHs? And how their orientation, with respect to the observer's line of sight, enters this challenging equation? 

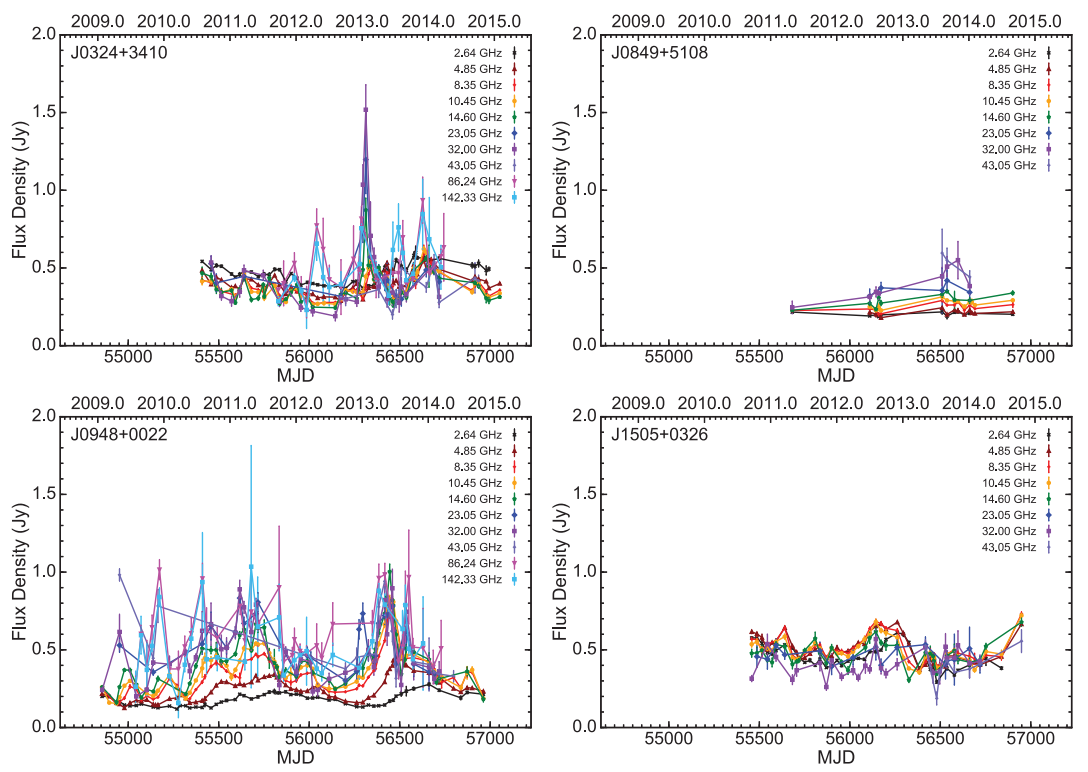

Figure 1. Updated radio light curves of $\gamma$ NLS1s between 2.6 and $42 \mathrm{GHz}$, until 2015 January. Where available, data at 86 and $142.33 \mathrm{GHz}$ are also shown.

In the following, we present our radio monitoring of $\gamma$ NLS1s using primarily the 100$\mathrm{m}$ telescope at Effelsberg and the IRAM 30-m telescope, while focusing on latest results. Furthermore, we focus on two $\gamma \mathrm{NLS} 1 \mathrm{~s}$ which have been studied in detail: (i) RX J2314.9+2243 with dedicated multi-wavelength monitoring, and (ii) $1 \mathrm{H} 0323+342$ with the help of high-resolution very-long-baseline interferometry (VLBI).

\section{Radio monitoring of NLS1 galaxies}

Our monthly monitoring of the jet emission from $\gamma$-ray-emitting NLS1 galaxies constitutes the most comprehensive such program at $\mathrm{cm}$ and short $\mathrm{mm}$ wavelengths (Angelakis et al. 2015). Observations with the Effelsberg 100-m telescope cover eight bands between 2.64 and $43.05 \mathrm{GHz}$. Until 2014, the IRAM 30-m telescope provided coverage at 86.24 and $142.33 \mathrm{GHz}$. The data collected in more than five years is the longest data set with the widest frequency coverage available for these objects (see Fig. 1). It includes also radio polarisation monitoring at $2.64,4.85,8.35$, and $10.45 \mathrm{GHz}$, with measurements of its linear and circular components and EVPAs. The observed sample comprises the following sources: $1 \mathrm{H} 0323+342$ (J0324+3410), SBS 0846+513 (J0849+5108), PMN J0948+0022 (J0948+0022), and PKS 1502+036 (J1505+0326). Recently, three more $\gamma$ NLS1s were included, namely FBQS J1644+2619, SDSS J1222+0413, and B3 1441+476.

All sources show the typical blazar behaviour although with lower flux densities. $\gamma$ NLS1s are variable and flare often, but the events are characterized by smaller amplitude and shorter duration. However, more energetic outbursts can be seen from time to time which dominate a source's light curve. Their spectra exhibit intense evolution with time, and steep, flat or even inverted spectra can be seen at different epochs. This evolution occurs faster than in blazars and can be ascribed to evolving shocks. The estimates of their jet powers are comparable with the output of the least energetic blazars. Furthermore, their moderate $\mathrm{T}_{\mathrm{b}}$ and associated Doppler factors $(\delta \lesssim 10)$, point to mildly relativistic jets as the driver of their phenomenology (Angelakis et al. 2015). 
Most sources do not display detectable polarisation. However, $1 \mathrm{H} 0323+342$ is $\sim 5.5 \%$ linearly polarized, higher than what is typically observed in blazars (e.g. $\sim 3.5-5 \%$ Myserlis 2015). Its EVPA, at $\sim 45^{\circ}$, lies perpendicular to the jet orientation, suggesting a magnetic field orientation almost parallel to the jet. PKS $1502+036$ appears to be polarized at 8.35 $\mathrm{GHz}\left(\sim 2-3 \%\right.$ with its EVPA at $\left.\sim 55^{\circ}\right)$ during flaring periods. In the $\mathrm{R}$ band, $\gamma \mathrm{NLS} 1 \mathrm{~s}$ have been monitored with RoboPol since 2013 (e.g. Angelakis et al. 2016). Their mean linear polarisation ranges from less than $1 \%$ to up to $20 \%$.

\section{The curious case of RX J2314.9+2243}

This is a mildly radio-loud $(\mathrm{R}=10-20)$ NLS1 galaxy at a redshift $\mathrm{z}=0.1692$ with a $\mathrm{BH}$ mass of $8 \times 10^{7} \mathrm{M}_{\odot}$ and a ratio $L / L_{\mathrm{Edd}}=0.2$ (Komossa et al. 2006 ). What sets this source apart from the rest of the NLS1 population is its tentative detection at $\gamma$ rays by Fermi (L. Foschini, priv. comm.) and its steep radio spectrum $(\alpha=-0.76$; Komossa et al. 2015). The ongoing radio observations verify the steep spectrum with flux densities $\mathrm{S}_{4 \mathrm{GHz}}=10 \pm 2 \mathrm{mJy}$ and $\mathrm{S}_{8 \mathrm{GHz}}=6 \pm 1 \mathrm{mJy}$ on two occasions in 2014 October. To date, all $\gamma$-ray detected NLS1s have been associated with flat spectrum sources and RX J2314.9+2243 could be the first steep radio spectrum NLS1 to emit $\gamma$ rays.

Komossa et al. (2015) address the emission processes shaping the spectral energy distribution (SED) of RX J2314.9+2243. The source is a luminous infrared emitter with an SED showing a broad emission hump between IR and UV bands, that steepens in the UV. An unusual feature of the source, compared to other NLS1s with steep X-ray spectra (e.g. Boller et al. 1996; Grupe 2004; Zhou et al. 2006), is the flatness of its X-ray spectrum, hinting at jet activity. In the UV on the other hand, the spectrum is very steep but there is no evidence of optical extinction beyond what is expected from our Galaxy, as would be expected by the dusty environment of luminous infrared galaxies (LIRGs). Therefore the IR to UV emission is most likely of non-thermal (i.e. synchrotron) origin arising from the operating jet.

Another striking feature of RX J2314.9+2243 is its very broad O[III] $5007 \AA$ emission line component. This shows an unusually high kinematical blueshift of $1260 \mathrm{~km} \mathrm{~s}^{-1}$ that could be due to the presence of an outflow powered by a radio jet feedback, in a face-on orientation with respect to our line of sight (Komossa et al. 2015).

\section{Direct imaging of $1 \mathrm{H} 0323+342$ at $15 \mathrm{GHz}$}

$1 \mathrm{H} 0323+342$ is the most nearby radio-loud $(\mathrm{R} \sim 50) \gamma$-ray emitting NLS1 at $\mathrm{z}=0.0629$ (Zhou et al. 2007). It exhibits a high Eddington luminosity ratio $\left(L / L_{\mathrm{Edd}} \sim 0.1\right)$ with a low BH mass of $\sim 10^{7} \mathrm{M}_{\odot}$ confirmed by several independent estimates (Zhou et al. 2007; Yao et al. 2015; Wang et al. 2016; Landt et al. 2016). 1H 0323+342 is highly variable at radio bands ( $\mathrm{cm}$ to $\mathrm{mm}$; see Fig. 1) and is also a special case because of the morphology of its host galaxy which appears ring-like or as a one-armed spiral (Zhou et al. 2007; Antón et al. 2008; León Tavares et al. 2014).

We studied the parsec-scale structure and kinematics of the source employing highresolution VLBI images at $15 \mathrm{GHz}$ obtained in eight epochs from 2010 October until 2013 July†. Additionally, with our single-dish observations we infer the $\mathrm{T}_{\mathrm{b}}$ and $\delta$ for the source. This combination provides a good estimate for the viewing angle towards 1H $0323+342$ (see Karamanavis 2015, for first results).

$1 \mathrm{H} 0323+342$ shows a core-jet morphology characterized by the prominent core, a straight jet, and a total absence of emission for the receding side of the jet; i.e. no counterjet is detected. A stationary feature is seen close to the core while six components move

$\dagger$ Data from the MOJAVE monitoring program (Lister et al. 2009). 
along the relativistic jet. Five jet components show superluminal motion with velocities from $1 \mathrm{c}$ up to $7 \mathrm{c}$ (Karamanavis 2015; Fuhrmann et al. 2016).

The source shows fast variability seen with single-dish and VLBI monitoring alike. The flaring events are of low to mild amplitude, but more rapid compared to the typical long-term behavior of blazars. $1 \mathrm{H} 0323+342$ shows high brightness temperatures. While Angelakis et al. (2015) report a Doppler factor of 3.6 at $14.6 \mathrm{GHz}$, and a higher one $(\delta=4.3)$ at $2.64 \mathrm{GHz}$, we deduce a more extreme value of $\mathrm{T}_{\mathrm{b}}=5.7 \times 10^{12} \mathrm{~K}$ and an associated Doppler factor of 5.2 , from the most rapid and largest flux density variation. Combining the knowledge on the source kinematics (i.e. the speeds of jet components) with the Doppler factors inferred from the observed variability, allowed us, under the assumption of causality, to constrain the viewing angle towards the source. The range of plausible values is $\theta \leqslant 4^{\circ}-13^{\circ}$ (Karamanavis 2015; Fuhrmann et al. 2016). The smaller value of this range is consistent with viewing angles typical of blazars, but nevertheless a larger $\theta$ with the respect to the observer's line of sight cannot be excluded.

\section{Concluding remarks}

The remarkable characteristics of NLS1 galaxies separate them as prominent class of AGN and made them, in recent years, the focal point of intensive research. These systems accrete matter at a rate near the Eddington limit and rapidly grow the low-mass black holes driving them. The study of the small population of radio-loud and gamma-ray detected NLS1s probes a parameter space not constrained by classical blazars and can provide a deeper understanding on the formation and evolution of powerful radio jets and the interplay with their complex environments.

\section{References}

Abdo, A. A., Ackermann, M., Ajello, M., et al. 2009a, ApJ, 707, 727

Abdo, A. A., Ackermann, M., Ajello, M., et al. 2009b, ApJ, 707, L142

Angelakis, E., Fuhrmann, L., Marchili, N., et al. 2015, A\&SA, 575, A55

Angelakis, E., Hovatta, T., Blinov, D., et al. 2016, MNRAS, 463, 3365

Antón, S., Browne, I. W. A., \& Marchã, M. J. 2008, A\&A, 490, 583

Boller, T., Brandt, W. N., \& Fink, H. 1996, A\& $A, 305,53$

Boroson, T. A. \& Green, R. F. 1992, ApJS, 80, 109

D'Ammando, F., Orienti, M., Finke, J., et al. 2013, MNRAS, 436, 191

Fuhrmann, L., Karamanavis, V., Komossa, S., et al. 2016, arXiv:1608.03232

Grupe, D. 2004, AJ, 127, 1799

Karamanavis, V. 2015, PhD thesis, University of Cologne, 2015

Komossa, S. 2008, in , Vol. 32, Revista Mexicana de Astronomia y Astrofisica Conference Series, 86

Komossa, S., Voges, W., Xu, D., et al. 2006, AJ, 132, 531

Komossa, S., Xu, D., Zhou, H., Storchi-Bergmann, T., \& Binette, L. 2008, ApJ, 680, 926

Komossa, S., Xu, D., Fuhrmann, L., et al. 2015, A\&A, 574, A121

Landt, H., Ward, M. J., Balokovic, M., et al. 2016, arXiv:1609.08002

León Tavares, J., Kotilainen, J., Chavushyan, V., et al. 2014, ApJ, 795, 58

Lister, M. L., Aller, H. D., Aller, M. F., et al. 2009, AJ, 137, 3718-3729

Myserlis, I. 2015, PhD thesis, University of Cologne, 2015

Osterbrock, D. E. \& Pogge, R. 1985, ApJ, 297, 166

Wang, F., Du, P., Hu, C., et al. 2016, ApJ, 824, 149

Yao, S., Yuan, W., Komossa, S., et al. 2015, AJ, 150, 23

Zhou, H., Wang, T., Yuan, W., et al. 2006, ApJS, 166, 128

Zhou, H., Wang, T., Yuan, W., et al. 2007, ApJ, 658, L13 